\title{
Fermionic correlation functions from the staggered Schrödinger functional
}

\author{
Paula Pérez Rubio*i \\ Instituto de Física Teórica UAM-CSIC, 28049 Cantoblanco, Spain, \\ and \\ School of Mathematics, Trinity College, Dublin 2, Ireland. \\ E-mail: perez@maths.tcd.ie

\section{Stefan Sint} \\ School of Mathematics, Trinity College, Dublin 2, Ireland \\ E-mail: sint@maths.tcd.ie
}

We consider the Schrödinger functional with staggered one-component fermions on a fine lattice of size $(L / a)^{3} \times(T / a)$ where $T / a$ must be an odd number. In order to reconstruct the fourcomponent spinors, two different set-ups are proposed, corresponding to the coarse lattice having size $(L / 2 a)^{3} \times\left(T^{\prime} / 2 a\right)$, with $T^{\prime}=T \pm a$. The continuum limit is then defined at fixed $T^{\prime} / L$. Both cases have previously been investigated in the pure gauge theory. Here we define fermionic correlation functions and study their approach to the continuum limit at tree-level of perturbation theory.

The XXVI International Symposium on Lattice Field Theory

July 14 - 19, 2008

Williamsburg, Virginia, USA

\footnotetext{
*Speaker.

${ }^{\dagger}$ This research was supported in part by the Universidad Autónoma de Madrid and in part by Trinity College Dublin.
} 


\section{Introduction}

Establishing a quantitative connection between the low energy and the perturbative regimes of QCD is one of the primary tasks for any attempt to solve QCD quantitatively. For lattice QCD, the main problem consists in the large scale differences involved, which cannot be resolved on a single finite lattice. A solution to this "non-perturbative renormalisation problem" has been proposed a while ago [1] and amounts to apply recursive finite size scaling techniques to the renormalised parameters and operators in a suitable renormalisation scheme. The Schrödinger functional (SF) [2, $3]$ gives rise to a class of such schemes with a number of technical advantages. In QCD with zero and two quark flavours, the running coupling $[2,4,6,7]$ and quark masses[5, 8, 9], as well as a range of composite operators have been studied. Note that the final results are obtained in the continuum limit and thus independent of the details of the lattice regularisation. Most results have been obtained using the implementation of the SF in QCD with Wilson type quarks [3]. However, for applications to QCD with four quark flavours, or for QCD like theories with multiples of four fermion flavours staggered fermions appear to be a natural alternative. Interesting universality tests could be devised and one may expect a better control over the continuum limit. Hence, in view of applications to four-flavour QCD we here revisit the implementation of the Schrödinger functional for staggered quarks, which has previously been studied in [10,11]. Its applications have so far been limited to studies of the running coupling for QCD-like theories with eight, twelve and sixteen fermion flavours $[11,12]$. As noticed in $[10,11]$, the time extent of the lattice, $T / a$, needs to be odd with staggered quarks, whereas the spatial lattice directions must have even extent, $L / a$. This makes it impossible to set $L=T$ exactly, and one needs to deal with the resulting $\mathrm{O}(a)$ effects. In order to cancel those, Heller [11] proposed to average results for the gauge coupling obtained in two separate simulations with $T=L \pm a$. While this seems to work out for the SF coupling, at least to one-loop order in perturbation theory, it is less clear how to proceed in the case of fermionic correlation functions. In particular, one needs to discuss how to reconstruct the four-component spinors in both cases, only one of which was considered in [11]. Ideally, one would like to avoid the averaging procedure altogether, and it has been shown in the pure gauge theory how this can be achieved by redefining the approach to the continuum limit [13].

This writeup is organised as follows. We start by reviewing the basics of the Schrödinger Functional and the definition of fermionic correlation functions. Next we reconstruct the action in terms of the four-component spinors for both cases, $T^{\prime}=T \pm a$. A chiral rotation is then carried out to recover the standard Schrödinger Functional boundary conditions for the fermionic fields. We show the results of the computation of the correlation functions at tree level of perturbation theory and we finish with an outlook to future work.

\section{The Schrödinger Functional and correlation functions}

The Schrödinger Functional is the Euclidean path integral of QCD on a hyper cylinder as space-time manifold. Dirichlet boundary conditions are imposed at Euclidean times $x_{0}=0, T$, while all fields are $L$-periodic in the spatial directions. For the fermionic fields one sets

$$
\begin{array}{ll}
\left.P_{+} \psi(y)\right|_{y_{0}=0}=\rho(\mathbf{y}) & \left.P_{-} \psi(y)\right|_{y_{0}=T}=\rho^{\prime}(\mathbf{y}), \\
\left.\bar{\psi}(y) P_{-}\right|_{y_{0}=0}=\bar{\rho}(\mathbf{y}) & \left.\bar{\psi}(y) P_{+}\right|_{y_{0}=T}=\bar{\rho}^{\prime}(\mathbf{y}),
\end{array}
$$


where $P_{ \pm}=\frac{1}{2}\left(1 \pm \gamma_{0}\right)$. Using a continuum notation, the spatial gauge field components satisfy the conditions,

$$
\left.A_{k}(y)\right|_{y_{0}=0}=\left.C_{k} \quad A_{k}(y)\right|_{y_{0}=T}=C_{k}^{\prime} .
$$

The Schrödinger Functional can then be regarded as a functional of the boundary fields,

$$
\mathscr{Z}\left[C, C^{\prime}, \rho, \rho^{\prime}, \bar{\rho}, \bar{\rho}^{\prime}\right]=\int \mathscr{D}[A, \psi, \bar{\psi}] e^{-S[A, \psi, \bar{\psi}]},
$$

and expectation values of any product of fields $\mathscr{O}$, are defined by,

$$
\langle\mathscr{O}\rangle=\left\{\frac{1}{\mathscr{Z}} \int D[A, \psi, \bar{\psi}] \mathscr{O} e^{-S[A, \psi, \bar{\psi}]}\right\}_{\rho=\rho^{\prime}=0 ; \bar{\rho}=\bar{\rho}^{\prime}=0} .
$$

Note that observables may contain quark and antiquark fields at the boundaries, by including derivatives with respect to the fermionic boundary fields, $\zeta(\mathbf{y})=\frac{\delta}{\delta \bar{\rho}(\mathbf{y})}, \bar{\zeta}(\mathbf{y})=-\frac{\delta}{\delta \rho(\mathbf{y})}$, and analogously for $\zeta^{\prime}(\mathbf{y}), \bar{\zeta}^{\prime}(\mathbf{y})$. Provided the gauge boundary fields are taken to be spatially constant, one may obtain gauge invariant quark bilinear sources at the boundaries, such as

$$
\mathscr{O}^{a}=\int \mathrm{d}^{3} \mathbf{y}^{\prime} \mathrm{d}^{3} \mathbf{y}^{\prime \prime} \bar{\zeta}\left(\mathbf{y}^{\prime}\right) \gamma_{5} \frac{1}{2} \tau^{a} \zeta\left(\mathbf{y}^{\prime \prime}\right)
$$

where $\tau^{a}$ is a flavour matrix and both the quark and anti-quark fields are projected to zero momentum. Using such sources the simplest fermionic correlation functions for the axial vector current and density take the form [14]

$$
f_{\mathrm{A}}^{a b}\left(y_{0}\right)=-\left\langle A_{0}^{a}(y) \mathscr{O}^{b}\right\rangle, \quad f_{\mathrm{P}}^{a b}\left(y_{0}\right)=-\left\langle P^{a}(y) \mathscr{O}^{b}\right\rangle, \quad f_{1}^{a b}=-\left\langle\mathscr{O}^{a} \mathscr{O}^{\prime} b\right\rangle
$$

Note that with an exact flavour symmetry all correlation functions would be proportional to $\delta^{a b}$.

\section{Reconstruction of the four component spinors}

\subsection{Case $T^{\prime}=T-a$}

This case is the one already discussed in[10,11]. The four-component spinors reside in a coarse lattice with lattice spacing $\bar{a}=2 a$. In Figure 1, the thin lines correspond to the fine lattice, and the dots represent the points of the coarse lattice where the reconstructed fermions live. The variable $y$ refers to the points in the coarse lattice, and $x$ to the fine lattice, and they are related by $x=2 y+a \xi$, with $\xi_{\mu}$ taking values in $\{0,1\}$. Introducing the transition fields $\chi_{\xi}(y)=\chi(x)$,

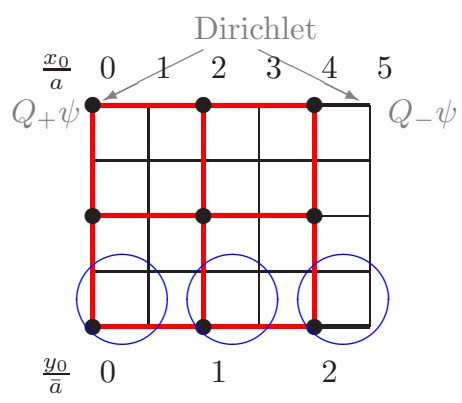

Figure 1: Reconstruction of the spinors on a $T=L+a$ lattice. 
$\bar{\chi}_{\xi}(y)=\bar{\chi}(x)$. the transformation is specified as

$$
\psi_{\alpha a}(y)=\frac{1}{4} \sum_{\xi}\left(\Gamma_{\xi}\right)_{\alpha a} \chi_{\xi}(y) \quad \bar{\psi}_{a \alpha}(y)=\frac{1}{4} \sum_{\xi} \bar{\chi}_{\xi}(y)\left(\Gamma_{\xi}\right)_{a \alpha},
$$

with $\Gamma_{\xi}=\frac{1}{2} \gamma_{0}^{\xi_{0}} \gamma_{1}^{\xi_{1}} \gamma_{2}^{\xi_{2}} \gamma_{3}^{\xi_{3}}$. In Figure 1, the one-component fermionic fields which constitute a reconstructed quark field are the ones contained in the circles. Note that the Dirichlet boundary conditions at $x_{0}=0, T$, imply a projection onto half of the components of the reconstructed quark field. Labelling the (hermitean) flavour matrices by their $\gamma$-matrix structure, e.g. $\tau_{\mu}=\gamma_{\mu}^{T}, \tau_{\mu 5}=$ $i\left(\gamma_{\mu} \gamma_{5}\right)^{T} \ldots$, and denoting the symmetric derivative by $\tilde{\partial}_{\mu}$ and the second derivative by $\Delta_{\mu}$, the boundary conditions read

$$
\begin{array}{ll}
Q_{+} \psi(0, \mathbf{y})=\hat{\rho}(\mathbf{y}), & Q_{-} \psi\left(T^{\prime}, \mathbf{y}\right)=\hat{\rho}^{\prime}(\mathbf{y}) \\
\bar{\psi}(0, \mathbf{y}) Q_{+}=\hat{\bar{\rho}}(\mathbf{y}), & \bar{\psi}\left(T^{\prime}, \mathbf{y}\right) Q_{-}=\hat{\bar{\rho}}^{\prime}(\mathbf{y})
\end{array}
$$

with projectors $Q_{ \pm}=\frac{1}{2}\left(1 \pm i \gamma_{0} \gamma_{5} \tau_{05}\right)$. For homogeneous boundary conditions, and with all fields at times $x_{0}<0$ and $x_{0}>T^{\prime}$ set to zero, the reconstructed action takes the form,

$$
S_{S Q}^{(s=-1)}=\bar{a}^{4} \sum_{y_{0}=0}^{T^{\prime}} \sum_{\mathbf{y}} \sum_{\mu} \bar{\psi}(y)\left[\gamma_{\mu} \tilde{\partial}_{\mu}+i \frac{\bar{a}}{2} \gamma_{5} \tau_{\mu 5} \Delta_{\mu}\right] \psi(y) .
$$

The usual SF boundary conditions can be recovered by performing a chiral rotation of the fermionic fields,

$$
\psi^{\prime}(y)=R(\alpha) \psi(y), \quad \bar{\psi}^{\prime}(y)=\bar{\psi}(y) R(\alpha), \quad R(\alpha)=\exp \left(i \frac{\alpha}{2} \gamma_{5} \tau_{05}\right) .
$$

For $\alpha=\frac{\pi}{2}$ the boundary conditions become the usual ones (2.1), due to $R\left(\frac{\pi}{2}\right) Q_{ \pm} R^{-1}\left(\frac{\pi}{2}\right)=P_{ \pm}$. For homogeneous boundary conditions, the action in the standard SF basis takes the form,

$$
S_{S Q}^{(s=-1)}=\bar{a}^{4} \sum_{y=0}^{T^{\prime}} \sum_{\mathbf{y}} \bar{\psi}^{\prime}(y)\left[\sum_{k} \gamma_{k} \mathscr{D}_{k}+\gamma_{0} \tilde{\partial}_{0}+\frac{\bar{a}}{2} \Delta_{0}\right] \psi^{\prime}(y),
$$

with $\mathscr{D}_{k}=\tilde{\partial}_{k}+i \frac{\bar{a}}{2} \gamma_{k} \gamma_{5} \tau_{k 5} \Delta_{k}$.

3.2 Case $T^{\prime}=T+a$

Here, we distinguish two alternative ways of reconstructing the fermions, as illustrated in Figure 2. We have labelled the two reconstructions with $s=1^{ \pm}$, according to the sign in front of $\xi_{0}$ in Eq. (3.6).

$$
\begin{array}{cc}
s=1^{+} & \multicolumn{1}{c}{s=1^{-}} \\
x_{0}=2 y_{0}-a+a \xi_{0}, \quad \mathbf{x}=2 \mathbf{y}+a \boldsymbol{\xi}, & x_{0}=2 y_{0}-a \xi_{0}, \quad \mathbf{x}=2 \mathbf{y}+a \boldsymbol{\xi}, \\
\psi_{\alpha a}(y)=\frac{1}{4} \sum_{\xi}\left(\tilde{\Gamma}_{\xi}\right)_{\alpha a} \chi_{\xi}(y), & \psi_{\alpha a}(y)=\frac{1}{4} \sum_{\xi}\left(\Gamma_{\xi}\right)_{\alpha a} \chi_{\xi}(y), \\
\bar{\psi}_{a \alpha}(y)=-\frac{1}{4} \sum_{\xi} \bar{\chi}_{\xi}(y)\left(\tilde{\Gamma}_{\xi}^{\dagger}\right)_{a \alpha}, & \bar{\psi}_{a \alpha}(y)=\frac{1}{4} \sum_{\xi} \bar{\chi}_{\xi}(y)\left(\Gamma_{\xi}^{\dagger}\right)_{a \alpha} \\
\tilde{\Gamma}_{\xi}=\frac{1}{2}(-1)^{\xi_{0}} \gamma_{0}^{\xi_{0}}, \gamma_{1}^{\xi_{1}} \gamma_{2}^{\xi_{2}} \gamma_{3}^{\xi_{3}} . &
\end{array}
$$




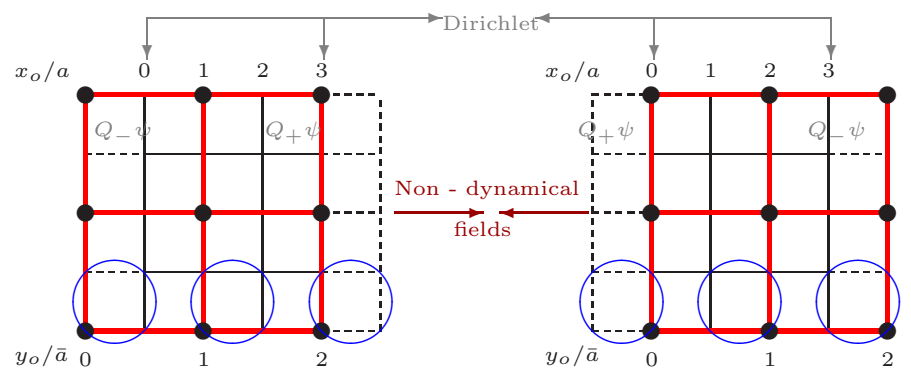

Figure 2: Reconstruction of the spinors on a $T=L-a$ lattice. Left $s=1^{+}$, right, $s=1^{-}$

The interpretation of Figure 2 is the same as Figure 1. Eqs. (3.6) specify how to reconstruct the four-component fermions in both cases. Depending on the reconstruction, the boundary conditions are different, and therefore the chiral rotations needed to restore the usual SF b.c.'s differ, too:

$$
\begin{array}{clrl}
s=1^{+} & \multicolumn{1}{c}{s=1^{-}} \\
Q_{-} \psi(0, \mathbf{y})=\hat{\rho}, \quad Q_{+} \psi(0, \mathbf{y})=\hat{\rho}^{\prime}, & Q_{+} \psi(0, \mathbf{y})=\hat{\rho}, \quad Q_{-} \psi(0, \mathbf{y})=\hat{\rho}^{\prime}, \\
\bar{\psi}(0, \mathbf{y}) Q_{-}=\hat{\bar{\rho}}, \quad \bar{\psi}^{\prime}(0, \mathbf{y}) Q_{+}=\hat{\bar{\rho}}^{\prime}, & \bar{\psi}(0, \mathbf{y}) Q_{+}=\hat{\bar{\rho}} \quad \bar{\psi}^{\prime}(0, \mathbf{y}) Q_{-}=\hat{\bar{\rho}}^{\prime}, \quad \\
\psi^{\prime}(y)=R\left(-\frac{\pi}{2}\right) \psi(y), \quad \bar{\psi}^{\prime}(y)=\bar{\psi}(y) R\left(-\frac{\pi}{2}\right), & \psi^{\prime}(y)=R\left(\frac{\pi}{2}\right) \psi(y), \quad \bar{\psi}^{\prime}(y)=\bar{\psi}(y) R\left(\frac{\pi}{2}\right) .
\end{array}
$$

However, once rotated to the standard SF basis, the action for both cases $s=1^{ \pm}$is the same,

$$
S_{S Q}^{(s=1)}=\bar{a}^{4} \sum_{y_{0}, \mathbf{y}} \bar{\psi}^{\prime}(y)\left[\sum_{k} \gamma_{k} \mathscr{D}_{k}+\gamma_{0} \tilde{\partial}_{0}-\frac{\bar{a}}{2} \Delta_{0}\right] \psi^{\prime}(y) .
$$

\subsection{Symmetries of the SF with staggered fermions}

The symmetries of the SF with staggered quarks have been summarised by Heller [11]. We here just wish to emphasise that the flavour and chiral symmetries refer to a particular basis. As we have seen, the boundary conditions may depend on the way the four-spinors are reconstructed. It is only after performing a chiral non-singlet rotation that the standard SF is recovered. In this basis, the usual axial U(1) symmetry of staggered quarks becomes a flavour symmetry. More precisely, the transformation

$$
\psi(y) \rightarrow e^{i \beta \gamma_{5} \tau_{5}} \psi(y), \quad \bar{\psi}(y) \rightarrow \bar{\psi}(y) e^{i \beta \gamma_{5} \tau_{5}},
$$

when rotated into the SF basis, becomes a continuous flavour symmetry with generator $\tau_{0}$,

$$
\psi^{\prime}(y) \rightarrow e^{i \beta \tau_{0}} \psi^{\prime}(y), \quad \bar{\psi}^{\prime}(y) \rightarrow \bar{\psi}^{\prime}(y) e^{-i \beta \tau_{0}} .
$$

Furthermore we note that spatial translations by a unit $a$ on the fine lattice.

$$
\psi(y) \rightarrow \tau_{k} \psi(y)+\bar{a} \tau_{k} Q_{+}(k) \partial_{k} \psi(y) \quad \bar{\psi}(y) \rightarrow \bar{\psi}(y) \tau_{k}+\bar{a} \bar{\psi}(y) \overleftarrow{\partial}_{k} \tau_{k} Q_{+}^{(k)},
$$

with $Q_{ \pm}^{(k)}=\frac{1}{2}\left(1 \pm \gamma_{k} \gamma_{5} \tau_{k 5}\right)$, correspond to a discrete subgroup of flavour symmetry in the continuum limit.

\section{Correlation functions at tree level}

To evaluate the correlation functions we first integrate over the quark fields. The expectation value assumes the form $\langle\mathscr{O}\rangle=\left\langle[\mathscr{O}]_{F}\right\rangle_{G}$, where \langle\rangle$_{G}$ denotes the gauge field average. We have determined the free quark propagator both analytically and numerically and may therefore compute the 
correlation functions to tree level. The remaining chiral symmetry of Eq. (3.9) becoming a flavour symmetry with generator $\tau_{0}$ in the standard SF basis, disconnected diagrams in the computation of $f_{A}, f_{P}, f_{1}$ are forbidden if we choose those flavour matrices which anticommute with $\tau_{0}$. For these matrices, the correlation functions $f_{A}^{a b}\left(y_{0}\right)$ reads,

$$
f_{\mathrm{A}}^{a b}\left(y_{0}\right)=\bar{a}^{6} \sum_{\mathbf{y}^{\prime}, \mathbf{y}^{\prime \prime}} \frac{1}{8}\left\langle\operatorname{tr}\left(\left[\zeta\left(\mathbf{y}^{\prime \prime}\right) \bar{\psi}^{\prime}(y)\right]_{F} \gamma_{0} \gamma_{5} \tau^{a}\left[\psi^{\prime}(y) \bar{\zeta}\left(\mathbf{y}^{\prime}\right)\right]_{F} \gamma_{5} \tau^{b}\right)\right\rangle_{G},
$$

and analogous expressions are obtained in the other cases. The continuum values of $f_{\mathrm{X}}^{a b}$ at tree level with vanishing background field take the form, $\delta^{a b} f_{\mathrm{X}}$, with

$$
f_{\mathrm{A}}\left(T^{\prime} / 2\right)=-\frac{N_{c}}{\cosh ^{2}(\sqrt{3} \theta)}, \quad f_{\mathrm{P}}\left(T^{\prime} / 2\right)=\frac{N_{c}}{\cosh (\sqrt{3} \theta)}, \quad f_{1}=\frac{N_{c}}{\cosh ^{2}(\sqrt{3} \theta)} .
$$

where $\theta$ is a phase factor coming from the generalised boundary conditions, i.e. $\psi(y+L \hat{k})=$ $e^{i \theta} \psi(y), \bar{\psi}(y+L \hat{k})=\bar{\psi}(y) e^{-i \theta}$. Including the correct tree level boundary counterterm, the results obtained are accurate up to $\mathrm{O}\left(a^{2}\right)$ for $f_{\mathrm{P}}, f_{1}$ and $\mathrm{O}(a)$ for $f_{\mathrm{A}}$.

\section{Fermionic $\mathbf{O}(a)$ improvement}

\subsection{Infinite volume}

Close to the continuum limit, the lattice theory may be described in terms of a local effective theory with action [15],

$$
S_{\text {eff }}=S_{0}+a S_{1}+a^{2} S_{2}+\ldots, \quad S_{k}=\int d^{4} y \mathscr{L}_{k}(y)
$$

The apparent $\mathrm{O}(a)$ contributions on the infinite lattice are fixed by the shift symmetry, since it is the combined expression $\mathscr{D}_{\mu} \gamma_{\mu}$ that is invariant under this transformation and not the usual kinetic term alone As was pointed out in [16], there are no invariant dimension 5 operators, so no counterterms can be added. The standard procedure to eliminate the apparent $\mathrm{O}(a)$ terms consists in defining improved field,

$$
\begin{aligned}
& \psi^{I}(y)=\psi(y)+\frac{\bar{a}}{4} \sum_{v}\left(Q_{+}^{(v)}-Q_{-}^{(v)}\right) \tilde{\partial}_{v} \psi(y), \\
& \bar{\psi}^{I}(y)=\bar{\psi}(y)+\frac{\bar{a}}{4} \sum_{v} \bar{\psi}^{\overleftarrow{\partial}}(y)\left(Q_{+}^{(v)}-Q_{-}^{(v)}\right)
\end{aligned}
$$

in terms of which one finds,

$$
S_{S Q}=\bar{a}^{4} \sum_{y \mu} \bar{\psi}^{I}(y) \gamma_{\mu} \tilde{\partial}_{\mu} \psi^{I}(y)+\mathrm{O}\left(a^{2}\right) .
$$

\section{$5.2 \mathrm{O}(a)$ effects from the boundaries}

In the SF framework, additional renormalisations and $\mathrm{O}(a)$ cutoff effects may arise from the very presence of the boundaries. Taking the symmetries into account, we arrive at the conclusion that there is only one possible dimension 3 operator, $\bar{\psi}^{\prime} \psi^{\prime}$. This is the same as encountered for Wilson quarks, and can thus be absorbed in a multiplicative renormalisation of the quark and antiquark fields at the boundaries. 
In the case of dimension 4 operators, we obtain again the same result as for Wilson quarks. However, when using the equations of motion, we here prefer a different choice for the counterterm action, namely

$$
\begin{gathered}
\delta S_{F, b}[U, \bar{\psi}, \psi]=\bar{a}^{4} \sum_{\mathbf{y}}\left\{\left(d_{1}-1\right)\left[\hat{\mathscr{O}}_{b, 1}+\hat{\mathscr{O}}_{b, 1}^{\prime}\right]+\left(d_{2}-1\right)\left[\hat{\mathscr{O}}_{b, 2}+\hat{\mathscr{O}}_{b, 2}^{\prime}\right]\right\}, \\
\hat{\mathscr{O}}_{b, 1}=\bar{\psi}^{\prime}(0, \mathbf{y}) P_{+} \gamma_{k} \mathscr{D}_{k} \psi^{\prime}(0, \mathbf{y}), \quad \hat{\mathscr{O}}_{b, 2}=\bar{\rho}(\mathbf{y}) \gamma_{k} \mathscr{D}_{k} \rho(\mathbf{y}), \\
\hat{\mathscr{O}}_{b, 1}^{\prime}=\bar{\psi}(T, \mathbf{y}) P_{-} \gamma_{k} \mathscr{D}_{k} \psi(T, \mathbf{y}), \quad \hat{\mathscr{O}}_{b, 2}^{\prime}=\bar{\rho}^{\prime}(\mathbf{y}) \gamma_{k} \mathscr{D}_{k} \rho^{\prime}(\mathbf{y}),
\end{gathered}
$$

The coefficients $d_{1,2}$ have a perturbation expansion in powers of $g_{0}^{2}$. We have determined $d_{1}$ at tree level,

\section{Conclusions}

$$
\left.d_{1}^{(0)}\right|_{T^{\prime}=T \pm a}=1 \pm \frac{1}{4}
$$

We have reconstructed the four-component spinors in the SF with staggered quarks, for both cases $T^{\prime}=T \pm a$, computed the free propagator and the correlation functions $f_{\mathrm{A}}, f_{\mathrm{P}}, f_{1}$ at tree level. The implementation of $\mathrm{O}(a)$ improvement is work in progress. Once it is fully understood, we plan to trace the running of the SF coupling and the quark mass in four-flavour QCD

\section{References}

[1] K. Jansen et al., Phys. Lett. B 372 (1996) 275 [arXiv:hep-lat/9512009].

[2] M. Lüscher, R. Narayanan, P. Weisz and U. Wolff, Nucl. Phys. B 384 (1992) 168 [arXiv:hep-lat/9207009].

[3] S. Sint, Nucl. Phys. B 421 (1994) 135.

[4] S. Sint and R. Sommer, Nucl. Phys. B 465 (1996) 71.

[5] S. Sint and P. Weisz [ALPHA collaboration], Nucl. Phys. B 545 (1999) 529.

[6] M. Lüscher, R. Sommer, P. Weisz and U. Wolff, Nucl. Phys. B 413 (1994) 481.

[7] M. Della Morte, R. Frezzotti, J. Heitger, J. Rolf, R. Sommer and U. Wolff [ALPHA Collaboration], Nucl. Phys. B 713 (2005) 378.

[8] S. Capitani, M. Luscher, R. Sommer and H. Wittig [ALPHA Collaboration], Nucl. Phys. B 544 (1999) 669.

[9] M. Della Morte, R. Hoffmann, F. Knechtli, J. Rolf, R. Sommer, I. Wetzorke and U. Wolff [ALPHA Collaboration], Nucl. Phys. B 729 (2005) 117.

[10] S. Miyazaki and Y. Kikukawa, arXiv:hep-lat/9409011.

[11] U. M. Heller, Nucl. Phys. B 504 (1997) 435; Nucl. Phys. Proc. Suppl. 63 (1998) 248.

[12] T. Appelquist, G. T. Fleming and E. T. Neil, Phys. Rev. Lett. 100, 171607 (2008).

[13] P. Perez-Rubio and S. Sint, PoS LAT2007, 249 (2007); P. Perez-Rubio, S. Sint, S. Takeda and $\mathrm{U}$. Wolff, in preparation.

[14] M. Lüscher, S. Sint, R. Sommer and P. Weisz, Nucl. Phys. B 478 (1996) 365.

[15] K. Symanzik, Nucl. Phys. B 226 (1983) 187.

[16] Y. b. Luo, Phys. Rev. D 55 (1997) 353. 